\title{
Atributos químicos e físicos de um Argissolo Vermelho-Amarelo em sistema integrado de produção agroecológica
}

\author{
Arcângelo Loss ${ }^{(1)}$, Marcos Gervasio Pereira(1), Nivaldo Schultz ${ }^{(1)}$, Lúcia Helena Cunha dos Anjos(1) \\ e Eliane Maria Ribeiro da Silva(2)
}

\begin{abstract}
(1) Universidade Federal Rural do Rio de Janeiro, Departamento de Solos, BR 465, Km 7, CEP 23890-000 Seropédica, RJ. E-mail: arcangeloloss@yahoo.com.br, gervasio@ufrrj.br, nsufrrj@yahoo.com.br, lanjos@ufrrj.br (2)Embrapa Agrobiologia, BR 465, Km 7, CEP 23890-000 Seropédica, RJ. E-mail: eliane@cnpab.embrapa.br
\end{abstract}

Resumo - O objetivo deste trabalho foi avaliar a influência de sistemas de manejo agroecológico sobre os atributos físicos e químicos de um Argissolo Vermelho-Amarelo. Foram selecionadas as seguintes áreas: preparo convencional (milho/feijão); plantio direto (berinjela/milho); consórcio maracujá/Desmodium sp.; área cultivada com figo; e sistema agroflorestal (SAF). Amostras indeformadas de solo foram coletadas em duas profundidades $(0-5$ e 5-10 cm) e em duas épocas (verão/2005 e inverno/2006). As propriedades edáficas analisadas foram: densidade do solo (Ds); volume total de poros; diâmetro médio ponderado (DMP) e diâmetro médio geométrico (DMG) de agregados; $\mathrm{pH}, \mathrm{Al}, \mathrm{Ca}+\mathrm{Mg}, \mathrm{K}, \mathrm{H}+\mathrm{Al}, \mathrm{P}$ e carbono orgânico total (COT). Os maiores valores de Ds, $\mathrm{P}$ e K foram verificados na área de figo. O sistema milho/feijão apresentou os menores valores de DMP e DMG. Os maiores valores de DMP e DMG foram observados nos sistemas maracujá/Desmodium e berinjela/milho. As diferenças entre os valores de COT foram maiores à profundidade de 5-10 cm. O SAF apresentou maiores percentuais de porosidade total. A análise de componentes principais mostrou que a área cultivada com figo está associada a maiores índices de fertilidade do solo.

Termos para indexação: agregação, densidade do solo, fertilidade do solo, manejo agroecológico, qualidade do solo.

\section{Chemical and physical attributes of an Udult soil in agroecological production systems}

\begin{abstract}
The objective of this study was to evaluate the influence of agroecological management systems on physical and chemical attributes of an Udult soil. The following areas were selected: conventional tillage (corn/beans); no tillage (eggplant/corn); consortium of passion fruit/Desmodium sp.; fig cultivation; and an agroforestry system (AFS). The undisturbed soil samples were taken from two layers $(0-5$ and $5-10 \mathrm{~cm})$ and in two periods, the summer of 2005 and the winter of 2006. The soil properties analyzed were: bulk density (Ds), total pore volume, mean weight diameter (MWD) and mean geometric diameter (MGD) of aggregates, pH, $\mathrm{Al}, \mathrm{Ca}+\mathrm{Mg}, \mathrm{K}, \mathrm{H}+\mathrm{Al}, \mathrm{P}$, and total organic carbon (TOC). The highest values of $\mathrm{Ds}, \mathrm{P}$ and $\mathrm{K}$ were observed in the fig area. The system with corn/beans had the lowest MWD and MGD values. The highest values of MWD and MGD were observed in the passion fruit/Desmodium and eggplant/corn systems. Differences in TOC were greater at the 5-10 cm depth. Soil in the AFS had the highest percentage of total porosity. The analysis of main components showed that the area cultivated with fig had the highest soil fertility.
\end{abstract}

Index terms: aggregation, bulk density, soil fertility, agroecological management, soil quality.

\section{Introdução}

O desenvolvimento agrícola envolve, inevitavelmente, certo grau de transformação física das paisagens e a artificialização dos ecossistemas. É essencial, portanto, conhecer estratégias que enfatizem procedimentos que levem a um desenvolvimento ecologicamente sustentável, ou seja, novos formatos tecnológicos compatíveis com a manutenção e recuperação do meio ambiente e a produção de alimentos confiáveis (Altieri \& Nicholls, 2000).

A agricultura orgânica surge como uma forma de amenizar esses problemas, já que nesta forma de cultivo várias práticas conservacionistas são usadas (Espindola, 2001). O uso de matéria orgânica, de origem vegetal ou animal, tem promovido melhorias

Pesq. agropec. bras., Brasília, v.44, n.1, p.68-75, jan. 2009 
nas propriedades edáficas do solo sob manejo orgânico de produção (Cardozo et al., 2008; Loss, 2008).

Há diversas técnicas de manejo adotadas pela agricultura orgânica, algumas das quais modificam positivamente as propriedades do solo, principalmente em agroecossistemas com culturas perenes. Exemplos dessas técnicas são: a manutenção da cobertura permanente do solo; a integração da adubação orgânica e verde; o controle da erosão, por meio do estabelecimento de curvas em nível; o terraceamento e as faixas de retenção; e o cultivos mínimo, em faixa ou bordadura. Com esse conjunto de práticas, procurase preparar e cultivar o solo de modo a evitar maior destruição dos agregados e a inversão das suas camadas, contrariamente ao que ocorre, em geral, no preparo convencional (Theodoro et al., 2003).

O Sistema Integrado de Produção Agroecológica (SIPA), área experimental da Embrapa Agrobiologia destinada a estudos em agricultura orgânica, destacase na identificação de cultivares adaptadas a sistemas orgânicos, no desenvolvimento de substratos apropriados para a produção de mudas, na adequação do uso de leguminosas para adubação verde, e no ajuste da técnica de plantio direto na agricultura orgânica. Entretanto, são ainda poucos os estudos realizados, e é recente a geração de conhecimento científico no que diz respeito às modificações promovidas nas propriedades físicas e químicas do solo decorrentes do manejo agroecológico com frutíferas e oleráceas.

O objetivo deste trabalho foi avaliar a influência de sistemas de manejo agroecológico sobre os atributos físicos e químicos de um Argissolo Vermelho-Amarelo.

\section{Material e Métodos}

O estudo foi realizado na área do Sistema Integrado de Produção Agroecológica (SIPA), implantado em 1993, em uma área de 59 ha. A área está localizada na Embrapa Agrobiologia, Seropédica, RJ (22\%45'S, $43^{\circ} 41^{\prime} \mathrm{W}$, à altitude de $\left.33 \mathrm{~m}\right)$. O clima é do tipo Aw, conforme a classificação de Köppen. O solo é do tipo Argissolo Vermelho-Amarelo (Embrapa Solos, 2006), com textura franco-arenosa, com valores médios de 784,168 e $48 \mathrm{~g} \mathrm{~kg}^{-1}$, à profundidade de $0-5 \mathrm{~cm}$, e de 770,168 e $62 \mathrm{~g} \mathrm{~kg}^{-1}$ na camada 5-10 cm, para areia, argila e silte, respectivamente.

No SIPA, são desenvolvidos experimentos com oleráceas e frutíferas, para a identificação de cultivares adaptadas a sistemas orgânicos, adequação do uso de leguminosas para adubação verde, ajuste do sistema plantio direto sob agricultura orgânica, entre outros. Foram selecionadas cinco áreas experimentais com 0,12 ha: cultivo de figo (Ficus carica) de sete anos e entrelinhas com gramíneas (Paspalum notatum); consórcio maracujá (Passiflora edulis)/Desmodium sp., em área cultivada com maracujá desde 1996; cultivo de milho (Zea mays), no verão, e feijão (Phaseolus vulgaris), no inverno, em área com experimentos há oito anos com rotação de culturas de milho, feijão, quiabo, couve e berinjela, com revolvimento do solo (aração e gradagem); cultivo de berinjela (Solanum melongena), no verão, e milho, no inverno, com a mesma sequência de rotação de culturas e tempo de utilização da área de milho/feijão, entretanto, em plantio direto e em sistema agroflorestal (SAF), de cinco anos, com cultivo de espécies frutíferas e arbóreas.

As áreas de cultivo com berinjela/milho, maracujá e milho/feijão estavam separadas por meio de faixas de 1,5 m de largura, onde foram cultivadas plantas de palmito jussara (Euterpe oleracea). Nas áreas com cultivo de figo e no SAF, a faixa era formada por árvores de carambola (Averrhoa carambola), situadas entre as três primeiras áreas e delas separadas por um carreador de aproximadamente $4 \mathrm{~m}$ de largura.

O manejo da fertilidade do solo das áreas foi iniciado com a correção da acidez pela incorporação de calcário dolomítico, em quantidade baseada nos resultados de análises de solo de cada gleba. As hortaliças e os cereais foram regularmente adubados com esterco de aviário e esterco bovino, e forneceram o equivalente à dose de $100 \mathrm{~kg} \mathrm{ha}^{-1} \mathrm{de}$ N. As fruteiras receberam esses mesmos insumos, aplicados nas covas de plantio e, posteriormente, em cobertura. Além de estercos, foram fornecidos P e K às culturas, mediante a aplicação de farinha de ossos, termofosfato sílico-magnesiano e cinzas de lenha. O esterco de aviário utilizado apresentou, em média, os seguintes teores de nutrientes $\left(\mathrm{g} \mathrm{kg}^{-1}\right)$ : $\mathrm{N}, 37,25$; $\mathrm{Ca}, 50,03 ; \mathrm{Mg}, 6,23 ; \mathrm{P}, 22,68 ; \mathrm{K}, 23,93$. O esterco bovino apresentou, em média, os seguintes teores de nutrientes $\left(\mathrm{g} \mathrm{kg}^{-1}\right)$ : N, 15,20; Ca, 9,68; $\mathrm{Mg}, 3,43$; $\mathrm{P}, 2,24 ; \mathrm{K}, 5,80$. Esta caracterização dos adubos utilizados no SIPA é decorrente de um histórico de análises dos estercos, encontrado em vários trabalhos desenvolvidos no local (Ribas et al., 2003; César, 2004; Castro et al., 2005; Silva, 2006). 
Os sistemas agroecológicos, selecionados para este trabalho, apresentavam as formas de manejo e adubação, descritos a seguir. O SAF não recebeu nenhum tipo de adubação complementar, ou seja, o fornecimento de nutrientes foi decorrente do aporte e da decomposição do material vegetal das espécies presentes no sistema; a área sob cobertura de maracujá, consorciada com Desmodium sp., teve as covas adubadas com esterco bovino, no momento do plantio, e recebeu duas adubações de cobertura com cama-de-aviário por ano; as áreas com cultivo de figo, milho/feijão e berinjela/ milho receberam adubação com cama-de-aviário no momento do plantio e, depois, em cobertura, de acordo com as necessidades de cada cultura.

$\mathrm{Na}$ área com plantio de figo, anteriormente a este trabalho, havia sido realizado consórcio da frutífera com crotalária (Crotalaria juncea) e siratro (Macroptilium atropurpureum). Na época deste trabalho havia apenas cobertura morta, composta por resíduos vegetais provenientes do corte da grama batatais (Paspalum notatum), que é mais largamente utilizada na estação chuvosa.

Anteriormente ao plantio de milho/feijão e berinjela/ milho, foram plantadas mucuna-cinza (Mucuna pruriens), mucuna-anã (Mucuna deeringiana), Crotalaria spectabilis e Crotalariajuncea, consorciadas com a cultura principal. Entretanto, no momento das coletas, essas leguminosas não estavam presentes na área.

A coleta das amostras de solo foi realizada em duas ocasiões: a primeira, no início do verão de 2005 (novembro), e a segunda no início do inverno de 2006 (junho). Todas as áreas avaliadas apresentavam relevo plano, e a coleta foi realizada em zigue-zague, nas entrelinhas das culturas, para não comprometer o sistema radicular. Para a avaliação das propriedades químicas do solo, foram coletadas cinco amostras compostas, formadas por três amostras simples, com cinco repetições por área. Para as propriedades físicas, foram coletadas cinco amostras simples indeformadas, em cada área, com auxílio de anel de Kopecky (diâmetro de 4,10 cm, altura de 3,40 cm); as amostragens foram realizadas nas profundidades de $0-5$ e 5-10 cm. Nesses mesmos locais, foram coletados torrões para a análise da estabilidade dos agregados.

As propriedades físicas avaliadas foram: densidade do solo (Ds), pelo método do anel volumétrico; o volume total de poros (VTP, \%); e a estabilidade de agregados via úmida, por meio dos índices de diâmetro médio ponderado (DMP) e diâmetro médio geométrico (DMG), conforme Embrapa (1997).

O diâmetro médio ponderado (DMP) foi calculado de acordo com a seguinte expressão:

$\mathrm{DMP}=\sum_{\mathrm{x}=1}$ xiyi, em que: $\mathrm{i}$ é $\mathrm{o}$ intervalo de classe $\stackrel{x}{8} \mathrm{~mm} \geq X>2 \mathrm{~mm}$, de $2 \mathrm{~mm} \geq X>1 \mathrm{~mm}$, de $1 \mathrm{~mm} \geq X>0,5 \mathrm{~mm}$, de $0,5 \mathrm{~mm} \geq X>0,25 \mathrm{~mm}$ e de $0,25 \mathrm{~mm} \geq X>0,105 \mathrm{~mm}$; xi é o diâmetro do centro de classe $(\mathrm{mm})$; yi é a razão entre a massa de agregados dentro da classe xi e a massa total de agregados.

O diâmetro médio geométrico (DMG) foi calculado de acordo com a seguinte expressão:

$\mathrm{DMG}=\exp \left\{\left(\sum w i 1 \mathrm{n} x \mathrm{i}\right) / \Sigma\right.$ wi $\}$, em que: wi é o peso dos agregados de cada centro de classe (g); ln é o logaritmo natural de xi; xi é o diâmetro do centro de classe (mm).

A caracterização química consistiu na determinação do $\mathrm{pH}$ em $\mathrm{H}_{2} \mathrm{O}$, relação da suspensão solo:água de

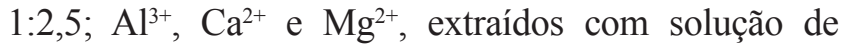
$\mathrm{KCl} 1 \mathrm{~mol} \mathrm{~L}^{-1}$, na proporção solo:solução de 1:10; $\mathrm{K}^{+}$e $\mathrm{P}$, extraídos com solução de $\mathrm{HCl} 0,05 \mathrm{~mol} \mathrm{~L}^{-1} \mathrm{e}$ $\mathrm{H}_{2} \mathrm{SO}_{4} 0,0125 \mathrm{~mol} \mathrm{~L}^{-1}$, na proporção solo:solução 1:10, em que o $\mathrm{K}$ foi determinado por fotometria de chama e o P por colorimetria, após a redução do complexo fosfomolibídico com ácido ascórbico, em presença de sal de bismuto; carbono orgânico total (COT), determinado pela oxidação da matéria orgânica pelo dicromato de potássio $0,2 \mathrm{~mol} \mathrm{~L}^{-1}$, em meio sulfúrico, e titulação pelo sulfato ferroso amoniacal $0,1 \mathrm{~mol} \mathrm{~L}^{-1}$, e $\mathrm{H}+\mathrm{Al}$ extraído com solução de acetato de cálcio $1 \mathrm{~mol} \mathrm{~L}^{-1}$, ajustada a pH 7, na proporção 1:15, segundo metodologia da Embrapa (1997). A partir dos resultados, calcularam-se a soma das bases trocáveis (valor $\mathrm{S}$ ) e a capacidade de troca catiônica (valor T).

Os dados das propriedades edáficas, para cada profundidade, foram submetidos à avaliação da normalidade e da homogeneidade das variâncias. Não houve necessidade de transformação dos dados. Posteriormente, os resultados foram submetidos à análise de variância com aplicação do teste $\mathrm{F}$, entre as áreas (milho/feijão, maracujá, SAF, figo e berinjela/ milho) e entre as estações (verão e inverno), ambas com cinco repetições. Em seguida, os valores médios foram comparados entre si pelo teste LSD a 5\% de probabilidade, pelo SAEG (Sistema de Análise Estatística e Genética), desenvolvido pela Universidade Federal de Viçosa. 
Realizou-se, também, a análise de componentes principais (ACP), por meio do SAS (SAS, 1999), para a camada de $0-10 \mathrm{~cm}$, cujas variáveis selecionadas foram as que apresentaram correlações de Pearson maiores que 0,7 entre si, relacionadas a seguir: VTP\%, DMP, DMG, Ds, COT, K, P, valor $\mathrm{S}$, valor T e $\mathrm{Ca}+\mathrm{Mg}$. A ACP é utilizada para reduzir as dimensões dos dados e, consequentemente, facilitar a análise por meio do gráfico do círculo de correlações (Herlihy \& McCarthy, 2006).

Os componentes principais, dispostos num espaço de duas dimensões, representam variabilidade suficiente para indicar algum padrão a ser interpretado. Autovetor é o valor que representa o peso de cada variável, em cada componente (eixos), e funciona como coeficiente de correlação que varia de $-1 \mathrm{a}+1$. As variáveis com elevado autovetor, no primeiro eixo, tendem a ter autovetor inferior no segundo eixo (Gomes et al., 2004; Tino, 2005).

\section{Resultados e Discussão}

Os menores valores de densidade do solo (Ds) foram verificados na camada de $0-5 \mathrm{~cm}$, sob todas as áreas das coberturas vegetais e sistemas de cultivo, quando comparados à camada de 5-10 cm (Tabela 1). Esse fato é decorrente da constante adição de matéria orgânica e do menor revolvimento a que as áreas são submetidas (Silva et al., 2006; Silveira Neto et al., 2006; Araújo et al., 2007).

O valor crítico de Ds para culturas comerciais, proposto por Reinert \& Reichert (2001) para solos com textura arenosa, com menos de $20 \%$ argila, é de $1,65 \mathrm{Mg} \mathrm{m}^{-3}$. No presente trabalho, foram verificados teores de argila inferiores a $20 \%$, e de areia acima de $75 \%$,

Tabela 1. Densidade do solo $\left(\mathrm{Mg} \mathrm{m}^{-3}\right)$ em consequência da cobertura vegetal, avaliada em amostras de solo coletadas nas profundidades de $0-5$ e 5-10 $\mathrm{cm}$, no verão e no inverno ${ }^{(1)}$.

\begin{tabular}{|c|c|c|c|c|}
\hline \multirow[t]{2}{*}{ Cobertura vegetal } & Verão & Inverno & Verão & Inverno \\
\hline & \multicolumn{2}{|c|}{$0-5 \mathrm{~cm}$} & \multicolumn{2}{|c|}{$5-10 \mathrm{~cm}$} \\
\hline Milho/feij & $1,33 \mathrm{BC}^{\mathrm{ns}}$ & $1,43 \mathrm{C}^{\mathrm{ns}}$ & $1,43 \mathrm{Ab}$ & $1,50 \mathrm{Ba}$ \\
\hline Maracujá & $1,35 \mathrm{~B}^{\mathrm{ns}}$ & $1,46 \mathrm{~B}^{\mathrm{ns}}$ & $1,42 \mathrm{~A}^{\mathrm{ns}}$ & $1,49 \mathrm{~B}^{\mathrm{ns}}$ \\
\hline SAF & $1,32 \mathrm{C}^{\mathrm{ns}}$ & $1,42 \mathrm{C}^{\mathrm{ns}}$ & $1,34 \mathrm{~B}^{\mathrm{ns}}$ & $1,43 C^{\mathrm{ns}}$ \\
\hline Figo & $1,38 \mathrm{Ab}$ & 1,48 & $1,43 \mathrm{~A}^{\mathrm{ns}}$ & $1,56 \mathrm{~A}^{\mathrm{ns}}$ \\
\hline Berinjela/milho & $1,35 \mathrm{Bb}$ & $1,48 \mathrm{Aa}$ & $1,45 \mathrm{Ab}$ & $1,47 \mathrm{Ba}$ \\
\hline
\end{tabular}

${ }^{(1)}$ Médias seguidas de letras maiúsculas iguais nas colunas não diferem entre si pelo teste LSD, a 5\% de probabilidade; médias seguidas de letras minúsculas iguais nas linhas, em cada camada, não diferem entre si, pelo

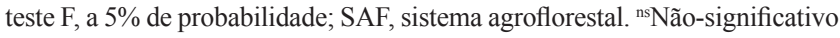
pelo teste $\mathrm{F}$, a $5 \%$ de probabilidade, entre as estações. e o maior valor de Ds $\left(1,56 \mathrm{Mg} \mathrm{m}^{-3}\right)$ foi encontrado na área de figo, na estação do inverno (Tabela 1). Portanto, os valores de Ds encontrados no SIPA não restringem o desenvolvimento do sistema radicular das culturas.

Os menores valores de porosidade (Tabela 2) foram verificados, de maneira geral, nas áreas que apresentaram os maiores valores de Ds (Tabela 1). Entre os valores de VTP, destaca-se a área de SAF com os maiores valores nas duas estações, na camada de 5-10 cm. Esses resultados são corroborados pelos baixos valores de Ds. Resultados semelhantes foram observados por Silva et al. (2006), que constataram maiores valores de VTP e menores valores de Ds, em cultivo de banana, em sistema agroflorestal, em áreas de agricultura no entorno do Parque Estadual da Serra do Mar, Ubatuba, SP. Quanto aos valores de DMP e DMG, a área de milho/feijão diferiu das demais, com os menores valores nas duas profundidades e estações do ano (Tabela 3). Tal fato pode ser explicado pelo maior revolvimento dessa área, onde se utiliza o preparo convencional do solo, com aração e gradagem. Esses resultados corroboram os de Pinheiro et al. (2004), que verificaram menores modificações na agregação do solo, em áreas de cultivo de oleráceas cultivadas sob plantio direto.

A área com maracujá apresentou os maiores valores de DMP e DMG (0-5 cm) no verão (Tabela 4). Esse resultado pode ser decorrente do consórcio com a leguminosa Desmodium sp., associado à adubação orgânica, que torna disponíveis elementos como $\mathrm{N}$ e $\mathrm{C}$ e propicia melhor desenvolvimento radicular das culturas e, consequentemente, melhora a agregação do solo, culminando na formação de agregados de maior tamanho.

Tabela 2. Percentagem de volume total de poros (VTP) em consequência da cobertura vegetal, em amostras de solo coletadas nas profundidades de $0-5$ e $5-10 \mathrm{~cm}$, no verão e no inverno ${ }^{(1)}$.

\begin{tabular}{|c|c|c|c|c|}
\hline \multirow[t]{2}{*}{ Cobertura vegetal } & Verão & Inverno & Verão & Inverno \\
\hline & \multicolumn{2}{|c|}{$0-5 \mathrm{~cm}$} & \multicolumn{2}{|c|}{$5-10 \mathrm{~cm}$} \\
\hline Milho/feijão & $48 \mathrm{~A}^{\mathrm{ns}}$ & $45 \mathrm{~A}^{\mathrm{ns}}$ & $45 \mathrm{~B}^{\mathrm{ns}}$ & $41 C^{\mathrm{ns}}$ \\
\hline Maracujá & $47 \mathrm{~A}^{\mathrm{ns}}$ & $44 A^{\mathrm{ns}}$ & $46 \mathrm{Ba}$ & $43 \mathrm{Bb}$ \\
\hline SAF & 49Aa & $45 \mathrm{Ab}$ & 49Aa & $45 \mathrm{Ab}$ \\
\hline Figo & $44 \mathrm{Ba}$ & $42 \mathrm{Bb}$ & $43 C^{n s}$ & $40 C^{\mathrm{ns}}$ \\
\hline Berinjela/milho & $46 \mathrm{Aa}$ & $42 \mathrm{Bb}$ & $42 \mathrm{Ca}$ & $43 \mathrm{Ba}$ \\
\hline \multicolumn{5}{|c|}{$\begin{array}{l}\text { (1) Médias seguidas de letras maiúsculas iguais nas colunas não diferem } \\
\text { entre si pelo teste LSD, a } 5 \% \text { de probabilidade; médias seguidas de letra } \\
\text { minúsculas iguais nas linhas, em cada camada, não diferem entre si pelc } \\
\text { teste F, a } 5 \% \text { de probabilidade; SAF, sistema agroflorestal. ns Não-significativc } \\
\text { pelo teste F, a } 5 \% \text { de probabilidade, entre as estações. }\end{array}$} \\
\hline
\end{tabular}

Pesq. agropec. bras., Brasília, v.44, n.1, p.68-75, jan. 2009 
Ao estudar o efeito da presença de Desmodium ovalifolium, na pastagem de Brachiaria humidicola, em condições de clima tropical, Tarré et al. (2001) verificaram que a taxa de acumulação de $\mathrm{C}$ no solo dobrou na área onde houve esse consórcio. Este comportamento é consequente da contribuição do $\mathrm{N}$ pela leguminosa e da importância que esse elemento possui na atividade biológica e na estabilização da matéria orgânica do solo (Sisti et al., 2004).

$\mathrm{Na}$ profundidade de $5-10 \mathrm{~cm}$, a área de berinjela/ milho apresentou os maiores valores de DMP, nas duas estações (Tabela 3). Esse resultado pode estar associado ao uso de adubação verde em cultivos anteriores (Ribas et al., 2003) e está de acordo com os resultados

Tabela 3. Diâmetro médio ponderado (DMP) e diâmetro médio geométrico (DMG) de agregados do solo das áreas avaliadas, sob diferentes coberturas vegetais, nas profundidades de $0-5$ e $5-10 \mathrm{~cm}$, no verão e no inverno ${ }^{(1)}$.

\begin{tabular}{|c|c|c|c|c|}
\hline \multirow[t]{2}{*}{ Cobertura vegetal } & \multicolumn{2}{|c|}{ DMP (mm) } & \multicolumn{2}{|c|}{ DMG (mm) } \\
\hline & Verão & Inverno & Verão & Inverno \\
\hline & \multicolumn{4}{|c|}{$0-5 \mathrm{~cm}$} \\
\hline Milho/feijão & $2,009 \mathrm{Ca}$ & $1,161 \mathrm{Bb}$ & $0,854 \mathrm{Da}$ & $0,570 \mathrm{Cb}$ \\
\hline Maracujá & $3,820 \mathrm{Aa}$ & $3,095 \mathrm{Ab}$ & $2,707 \mathrm{Aa}$ & $1,535 \mathrm{Bb}$ \\
\hline SAF & $3,379 \mathrm{Ba}$ & $3,100 \mathrm{Ab}$ & $2,112 \mathrm{BCa}$ & $1,555 \mathrm{Bb}$ \\
\hline Figo & $3,632 \mathrm{ABa}$ & $3,151 \mathrm{Ab}$ & $2,359 \mathrm{ABa}$ & $1,805 \mathrm{Ab}$ \\
\hline \multirow[t]{2}{*}{ Berinjela/milho } & $3,389 \mathrm{Ba}$ & $3,151 \mathrm{Ab}$ & $1,770 C^{\mathrm{ns}}$ & $1,635 \mathrm{AB}^{\mathrm{ns}}$ \\
\hline & \multicolumn{4}{|c|}{$5-10 \mathrm{~cm}$} \\
\hline Milho/feijão & $1,550 \mathrm{Cb}$ & $1,886 \mathrm{Ca}$ & $0,630 \mathrm{Bb}$ & $0,849 \mathrm{Ba}$ \\
\hline Maracujá & $2,395 \mathrm{Bb}$ & $2,672 \mathrm{Ba}$ & $1,266 \mathrm{~A}^{\mathrm{ns}}$ & $1,312 \mathrm{~A}^{\mathrm{ns}}$ \\
\hline SAF & $2,455 \mathrm{Bb}$ & $2,621 \mathrm{Ba}$ & $1,320 \mathrm{~A}^{\mathrm{ns}}$ & $1,317 \mathrm{~A}^{\mathrm{ns}}$ \\
\hline Figo & $2,695 \mathrm{AB}^{\mathrm{ns}}$ & $2,686 \mathrm{~B}^{\mathrm{ns}}$ & $1,322 \mathrm{~A}^{\mathrm{ns}}$ & $1,280 \mathrm{~A}^{\mathrm{ns}}$ \\
\hline Berinjela/milho & $2,932 \mathrm{~A}^{\mathrm{ns}}$ & $2,848 \mathrm{~A}^{\mathrm{ns}}$ & $1,489 A^{\mathrm{ns}}$ & $1,452 \mathrm{~A}^{\mathrm{ns}}$ \\
\hline \multicolumn{5}{|c|}{$\begin{array}{l}{ }^{(1)} \text { Médias seguidas de letras maiúsculas iguais, nas colunas, em cada } \\
\text { camada, não diferem entre si pelo teste LSD, a } 5 \% \text { de probabilidade; } \\
\text { médias seguidas de letras minúsculas iguais, nas linhas, para DMP e DMG, } \\
\text { não diferem entre si pelo teste } \mathrm{F} \text {, a } 5 \% \text { de probabilidade; SAF, sistema } \\
\text { agroflorestal. ns Não-significativo pelo teste } \mathrm{F} \text {, a } 5 \% \text { de probabilidade, entre } \\
\text { as estações. }\end{array}$} \\
\hline
\end{tabular}

encontrados por Perin et al. (2002). Nas áreas de SAF e figo, também se fez uso de adubação verde, porém a área de berinjela/milho foi manejada com plantio direto (SPD), o que propicia a manutenção dos resíduos vegetais na superfície do solo, assim como os maiores valores de COT (Tabela 4). Resultados similares foram verificados por Souza Neto et al. (2008) em rotação de culturas em SPD.

Entre as estações, observaram-se maiores valores de DMP e DMG, na camada superficial na estação do verão, e à profundidade de $5-10 \mathrm{~cm}$ na estação do inverno (Tabela 3). Esses resultados são decorrentes dos maiores teores de COT encontrados nas diferentes estações e profundidades (Tabela 4). Pinheiro et al. (2004), Weidling et al. (2005) e Reichert et al. (2006) encontraram resultados semelhantes aos verificados neste trabalho.

Quanto à fertilidade do solo, verificaram-se valores de $\mathrm{pH}$ acima de 6,2 e 5,8, nas profundidades $0-5$ e 5-10 cm, respectivamente (Tabela 4). Os elevados valores de $\mathrm{pH}$ são decorrentes da ausência de $\mathrm{Al}^{3+} \mathrm{e}$ dos baixos valores de $\mathrm{H}+\mathrm{Al}$, verificados nas áreas de estudo. Resultados semelhantes foram relatados por Theodoro et al. (2003), ao avaliar alterações químicas de um Latossolo Vermelho cultivado com café e submetido a manejo orgânico. Os autores observaram valores de $\mathrm{pH}$ em torno de 6,78 , e de $\mathrm{H}+\mathrm{Al}$ próximos de $1,70 \mathrm{cmol}_{\mathrm{c}} \mathrm{kg}^{-1}$.

Os valores médios de $\mathrm{Ca}+\mathrm{Mg}, \mathrm{K}$ e $\mathrm{P}$, assim como os valores $\mathrm{S}$ e $\mathrm{T}$, de maneira geral, são considerados altos segundo De-Polli et al. (1988), para solos do Estado do Rio de Janeiro. Esses elevados valores são decorrentes do manejo adotado, pois na adubação orgânica, realizada no SIPA, utiliza-se cama-de-aviário, esterco bovino, adubações verdes, termofosfato e cinzas de

Tabela 4. Propriedades químicas do solo, avaliadas nas áreas sob as diferentes coberturas vegetais, nas camadas 0-5 e $5-10 \mathrm{~cm}$, no verão e no inverno ${ }^{(1)}$.

\begin{tabular}{|c|c|c|c|c|c|c|c|c|c|c|c|c|c|c|c|c|}
\hline \multirow{2}{*}{$\begin{array}{l}\text { Cobertura } \\
\text { vegetal } \\
\end{array}$} & \multicolumn{2}{|c|}{$\mathrm{pH}$} & \multicolumn{2}{|c|}{$\mathrm{H}+\mathrm{Al}\left(\mathrm{cmol}_{\mathrm{c}} \mathrm{kg}^{-1}\right)$} & \multicolumn{2}{|c|}{$\mathrm{Ca}+\mathrm{Mg}\left(\mathrm{cmol}_{\mathrm{c}} \mathrm{kg}^{-1}\right)$} & \multicolumn{2}{|c|}{$\mathrm{K}\left(\mathrm{cmol}_{\mathrm{c}} \mathrm{kg}^{-1}\right)$} & \multicolumn{2}{|c|}{$\mathrm{P}\left(\mathrm{mg} \mathrm{kg}^{-1}\right)$} & \multicolumn{2}{|c|}{$\operatorname{COT}\left(\mathrm{g} \mathrm{kg}^{-1}\right)$} & \multicolumn{2}{|c|}{ Valor S } & \multicolumn{2}{|c|}{ Valor T } \\
\hline & Verão & Inverno & Verão & Inverno & Verão & Inverno & Verão & Inverno & Verão & Inverno & Verão & Inverno & Verão & Inverno & Verão & Inverno \\
\hline & & & & & & & & & $0-5 \mathrm{~cm}$ & & & & & & & \\
\hline Milho/feijão & $6,77 \mathrm{Ab}$ & $7,00 \mathrm{Aa}$ & $1,70 \mathrm{Aa}$ & $1,20 \mathrm{Bb}$ & $5,46 \mathrm{Bb}$ & $6,48 \mathrm{Aa}$ & $0,32 \mathrm{C}^{\mathrm{ns}}$ & $0,32 C^{\mathrm{ns}}$ & $109,72 \mathrm{Bb}$ & $139,61 \mathrm{Ba}$ & $9,48 \mathrm{C}^{\mathrm{ns}}$ & $9,13 \mathrm{~B}^{\mathrm{ns}}$ & $5,87 \mathrm{Bb}$ & 6,91Aa & $7,58 \mathrm{~B}^{\mathrm{ns}}$ & $8,10 \mathrm{~B}^{\mathrm{ns}}$ \\
\hline Maracujá & $6,21 \mathrm{~B}^{\mathrm{ns}}$ & $6,48 \mathrm{~B}^{\mathrm{ns}}$ & $1,70 \mathrm{Ab}$ & $2,38 \mathrm{Aa}$ & $4,28 \mathrm{Cb}$ & $6,40 \mathrm{Aa}$ & $0,49 \mathrm{Ba}$ & $0,29 \mathrm{Cb}$ & $92,11 \mathrm{Ca}$ & $57,57 \mathrm{~Eb}$ & $9,07 \mathrm{C}^{\mathrm{ns}}$ & $8,77 \mathrm{~B}^{\mathrm{ns}}$ & $4,84 \mathrm{Cb}$ & $6,76 \mathrm{Aa}$ & $6,54 \mathrm{Cb}$ & $9,14 \mathrm{Aa}$ \\
\hline SAF & $6,53 \mathrm{Ab}$ & $6,75 \mathrm{Ba}$ & $1,69 \mathrm{Aa}$ & $1,40 \mathrm{Bb}$ & $4,70 C^{\mathrm{ns}}$ & $4,96 \mathrm{C}^{\mathrm{ns}}$ & $0,22 \mathrm{Db}$ & $0,25 \mathrm{Ca}$ & $65,02 \mathrm{Db}$ & $96,93 \mathrm{Ca}$ & $9,44 C^{\mathrm{ns}}$ & $8,93 \mathrm{~B}^{\text {ns }}$ & $4,95 \mathrm{C}^{\mathrm{ns}}$ & $5,27 \mathrm{C}^{\mathrm{ns}}$ & $6,65 C^{\mathrm{ns}}$ & $6,67 C^{\text {ns }}$ \\
\hline Figo & $6,55 \mathrm{Ab}$ & $6,91 \mathrm{Aa}$ & $1,66 \mathrm{Aa}$ & $1,35 \mathrm{Bb}$ & $6,16 \mathrm{~A}^{\mathrm{ns}}$ & $5,66 \mathrm{~B}^{\mathrm{ns}}$ & $0,75 \mathrm{Ab}$ & $0,83 \mathrm{Aa}$ & $291,20 \mathrm{~A}^{\mathrm{ns}}$ & $287,47 \mathrm{~A}^{\mathrm{ns}}$ & $13,70 \mathrm{Aa}$ & $9,06 \mathrm{Bb}$ & $6,99 \mathrm{~A}^{\mathrm{ns}}$ & $6,56 \mathrm{~A}^{\mathrm{ns}}$ & $8,64 \mathrm{Aa}$ & $7,92 \mathrm{Bb}$ \\
\hline Beringela/milho & $6,65 \mathrm{Ab}$ & $7,10 \mathrm{Aa}$ & $1,37 \mathrm{~B}^{\mathrm{ns}}$ & $1,12 \mathrm{~B}^{\mathrm{ns}}$ & $5,30 \mathrm{~B}^{\mathrm{ns}}$ & $5,16 \mathrm{C}^{\mathrm{ns}}$ & $0,25 \mathrm{Db}$ & $0,56 \mathrm{Ba}$ & $110,11 \mathrm{Ba}$ & $87,46 \mathrm{Db}$ & $10,97 \mathrm{Ba}$ & $9,82 \mathrm{Ab}$ & $5,61 \mathrm{~B}^{\mathrm{ns}}$ & $5,78 \mathrm{~B}^{\mathrm{ns}}$ & $6,97 \mathrm{C}^{\mathrm{ns}}$ & $6,91 \mathrm{C}^{\mathrm{ns}}$ \\
\hline & & & & & & & & & $5-10 \mathrm{~cm}$ & & & & & & & \\
\hline Milho/feijão & $6,93 \mathrm{~A}^{\mathrm{ns}}$ & $7,08 \mathrm{~A}^{\mathrm{ns}}$ & $1,04 C^{\mathrm{ns}}$ & $1,05 \mathrm{D}^{\mathrm{ns}}$ & $5,38 \mathrm{Ab}$ & $6,46 \mathrm{Aa}$ & $0,40 \mathrm{Ba}$ & $0,29 \mathrm{Bb}$ & $95,52 \mathrm{Bb}$ & $109,94 \mathrm{Ba}$ & $8,89 \mathrm{Cb}$ & $10,34 \mathrm{Aa}$ & $5,87 \mathrm{Ab}$ & $6,84 \mathrm{Aa}$ & $6,91 \mathrm{Bb}$ & $7,88 \mathrm{Aa}$ \\
\hline Maracujá & $5,87 \mathrm{C}^{\mathrm{ns}}$ & $5,96 \mathrm{C}^{\mathrm{ns}}$ & $1,57 \mathrm{Bb}$ & $2,65 \mathrm{Aa}$ & $4,02 \mathrm{Cb}$ & $5,36 \mathrm{Ba}$ & $0,22 \mathrm{C}^{\mathrm{ns}}$ & $0,22 \mathrm{C}^{\mathrm{ns}}$ & $47,13 \mathrm{Ca}$ & $40,13 \mathrm{Db}$ & $7,42 \mathrm{Db}$ & $8,21 \mathrm{Ca}$ & $3,73 \mathrm{Db}$ & $5,66 \mathrm{Ca}$ & $5,29 \mathrm{Cb}$ & $8,30 \mathrm{Aa}$ \\
\hline SAF & $5,82 \mathrm{Cb}$ & $6,54 \mathrm{Ba}$ & $1,53 \mathrm{Ba}$ & $1,25 \mathrm{Cb}$ & $4,48 \mathrm{~B}^{\mathrm{ns}}$ & $4,52 \mathrm{C}^{\mathrm{ns}}$ & $0,11 \mathrm{Db}$ & $0,12 \mathrm{Da}$ & $42,80 \mathrm{Cb}$ & $80,08 \mathrm{Ca}$ & 7,04Eb & $8,05 \mathrm{CDa}$ & $4,63 C^{n s}$ & $4,71 \mathrm{D}^{\mathrm{ns}}$ & $6,16 \mathrm{~B}^{\mathrm{ns}}$ & $5,96 \mathrm{~B}^{\mathrm{ns}}$ \\
\hline Figo & $6,63 \mathrm{~B}^{\mathrm{ns}}$ & $6,81 \mathrm{~B}^{\text {ns }}$ & $2,53 \mathrm{Aa}$ & $1,65 \mathrm{Bb}$ & $5,30 \mathrm{~A}^{\mathrm{ns}}$ & $5,56 \mathrm{~A}^{\mathrm{ns}}$ & $0,58 \mathrm{Ab}$ & $0,68 \mathrm{Aa}$ & $158,15 \mathrm{~A}^{\mathrm{ns}}$ & $160,98 \mathrm{~A}^{\mathrm{ns}}$ & $11,49 \mathrm{Aa}$ & $7,84 \mathrm{Db}$ & $5,95 \mathrm{~A}^{\mathrm{ns}}$ & $6,32 \mathrm{~B}^{\mathrm{ns}}$ & $8,48 \mathrm{~A}^{\mathrm{ns}}$ & $7,97 \mathrm{~A}^{\mathrm{ns}}$ \\
\hline Beringela/milho & $6,60 \mathrm{~B}^{\mathrm{ns}}$ & $6,77 \mathrm{~B}^{\mathrm{ns}}$ & $1,43 \mathrm{~B}^{\mathrm{ns}}$ & $1,29 \mathrm{C}^{\mathrm{ns}}$ & $4,72 \mathrm{~B}^{\mathrm{ns}}$ & $4,64 C^{\mathrm{ns}}$ & $0,24 \mathrm{C}^{\mathrm{ns}}$ & $0,24 C^{\mathrm{ns}}$ & $95,77 \mathrm{Ba}$ & $85,39 \mathrm{Cb}$ & $9,53 \mathrm{~B}^{\text {ns }}$ & $9,32 \mathrm{~B}^{\mathrm{ns}}$ & $5,05 \mathrm{~B}^{\mathrm{ns}}$ & $4,97 \mathrm{D}^{\mathrm{ns}}$ & $6,48 \mathrm{~B}^{\mathrm{ns}}$ & $6,26 \mathrm{~B}^{\mathrm{ns}}$ \\
\hline
\end{tabular}


lenha. Essa adubação realizada com frequência está aumentando ou favorecendo os elevados níveis de nutrientes no solo.

Ao avaliar o efeito do cultivo do quiabeiro (Abelmoschus esculentus) consorciado com Crotalaria juncea, sob manejo orgânico no SIPA, na mesma área da cobertura de berinjela/milho, Ribas et al. (2003) também verificaram elevados valores para os parâmetros de fertilidade na camada de $0-20 \mathrm{~cm}$, com: $\mathrm{pH}, 6,6 ; \mathrm{Al}, 0 \mathrm{mmol}_{\mathrm{c}} \mathrm{kg}^{-1}$; Ca, $34 \mathrm{mmol}_{\mathrm{c}} \mathrm{kg}^{-1} ; \mathrm{Mg}$, $13 \mathrm{mmol}_{\mathrm{c}} \mathrm{kg}^{-1} ; \mathrm{K}, 2,3$ mmol $_{\mathrm{c}} \mathrm{kg}^{-1} ; \mathrm{P}, 106 \mathrm{mg} \mathrm{kg}^{-1}$.

As áreas sob cobertura de figo e de berinjela/milho apresentaram maiores teores de COT que as demais, nas duas profundidades, na estação do verão, com destaque para a área com figo, com os maiores teores de COT (Tabela 4). Tal resultado pode ser atribuído à cobertura morta presente no solo no momento da coleta, já que na área com berinjela/milho foi utilizada a rotação de culturas em SPD, o que permitiu a manutenção e o acúmulo de resíduos vegetais na superfície do solo. A cobertura morta utilizada na área de figo consistiu de material roçado da própria vegetação de gramíneas (Paspalum notatum) da área, prática essa observada apenas na estação do verão. Também se destacou a influência da adubação verde com leguminosas, em cultivos anteriores nessas áreas, de acordo com Ribas et al. (2003). Essa prática pode aumentar os teores de COT, quando os adubos verdes são introduzidos junto ao cultivo (Duda et al., 2003).

$\mathrm{Na}$ estação do inverno, os maiores teores de COT foram observados na área de berinjela/milho, na profundidade de $0-5 \mathrm{~cm}$, e nas áreas de milho/feijão e berinjela/milho, na profundidade de $5-10 \mathrm{~cm}$, (Tabela 4). Isso pode ser explicado pela ausência de cobertura morta na área com figo, e pela adoção do SPD na área com cobertura sob berinjela/milho, uma vez que, no momento da coleta do solo, a cultura do milho já havia sido colhida, e os resíduos culturais permaneceram na superfície, para novo plantio de berinjela sobre a palhada do milho. $\mathrm{Na}$ área de milho/ feijão, a incorporação da palhada de milho, para semeadura do feijão, pode ter beneficiado o aumento de COT. Esses resultados estão de acordo com os observados por Bertol et al. (2004).

$\mathrm{Na}$ profundidade de $5-10 \mathrm{~cm}$, foram encontradas as maiores variações nos teores médios de COT entre as áreas analisadas (Tabela 4), diferentemente da profundidade de $0-5 \mathrm{~cm}$, onde a adubação orgânica utilizada em todas as áreas homogeneizou os teores médios de COT - como notado nas áreas de milho/ feijão, maracujá e SAF, nas duas estações. Na profundidade de 5-10 cm, o manejo dado em cada área, associado à quantidade de resíduos vegetais oriundos de cada cultura e à textura (\% de areia) em cada área, influenciou os teores médios de COT, principalmente no verão, período com maior disponibilidade de água e temperaturas mais elevadas. Esse ambiente acarretou o aumento da velocidade das reações químicas no solo, com mineralização rápida da matéria orgânica do solo, o que resultou em teores de COT diferentes em cada área, como observado no verão, quando todos os sistemas apresentaram diferenças estatísticas entre si.

Quanto à análise de componentes principais (ACP), verificou-se que a distribuição das variáveis selecionadas apresentou variância acumulada de $72,97 \%$ para os eixos F1 e F2, sendo que o eixo F1 foi capaz de explicar $49,54 \%$ e o F2, $23,43 \%$ dessa variância (Figura 1).

Ao avaliar a influência da granulometria e da mineralogia sobre a retenção de $\mathrm{P}$, em Latossolos sob pastagens no Cerrado, Eberhardt et al. (2008) verificaram, por meio da ACP realizada sobre o

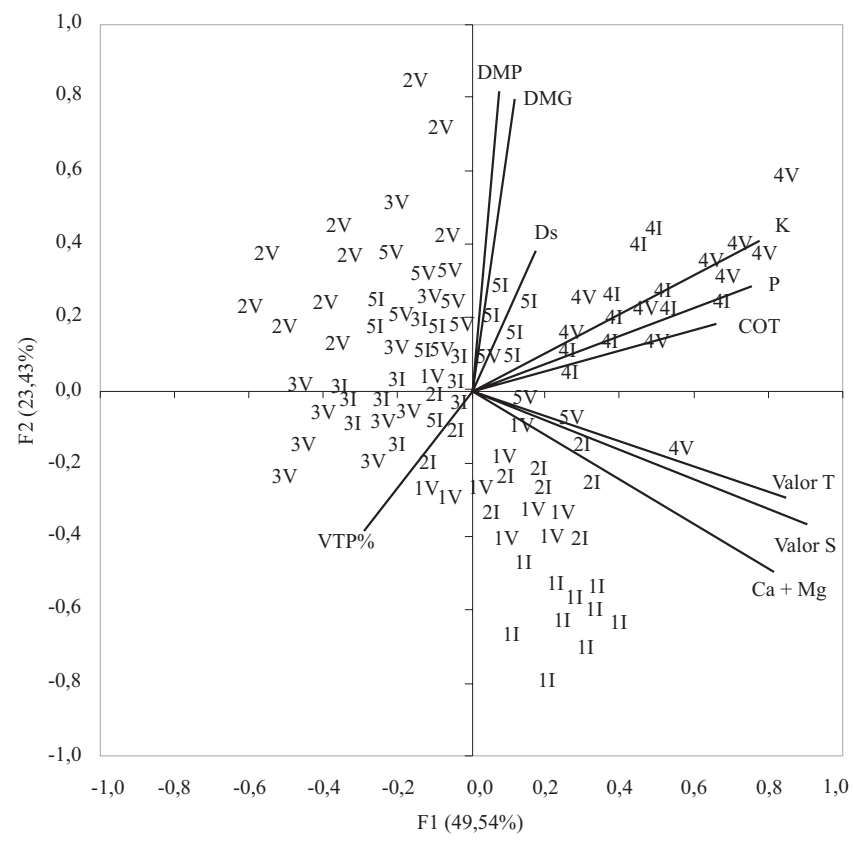

Figura 1. Diagrama de ordenação produzido por análise de componentes principais, das características físicas e químicas avaliadas em amostras de solo coletadas na camada de $0-10 \mathrm{~cm}$, em que I representa inverno; V, verão; 1, milho/feijão; 2, maracujá; 3, SAF; 4, figo; 5, berinjela/milho. 
conjunto de dados físicos, químicos e mineralógicos dos solos, que os dois primeiros eixos explicaram $56 \%$ da variabilidade total dos dados, dos quais $38 \%$ no eixo F1 e $18 \%$ no eixo F2. No presente trabalho, observou-se maior variância acumulada em função das variáveis selecionadas, cuja maior parte é explicada pelas variáveis químicas do solo (Figura 1).

$\mathrm{Na} \mathrm{ACP}$, as características do solo são representadas porsetas, queindicama direção do gradiente máximo,em que o comprimento da seta é proporcional à correlação da característica com os eixos e à sua importância na explicação da variância projetada em cada eixo (Theodoro et al., 2003). Neste sentido, destaca-se para correlação com o eixo $\mathrm{F} 1$, as propriedades químicas, em que $\mathrm{COT}$, $\mathrm{K}$ e $\mathrm{P}$ apresentaram autovetores positivos, e $\mathrm{T}, \mathrm{S}$ e $\mathrm{Ca}+\mathrm{Mg}$ apresentaram autovetores negativos. Também se observa a influência do VTP, no eixo F1, com autovetor negativo. No eixo F2, as propriedades físicas DMP, DMG e Ds apresentaram autovetores positivos (Figura 1).

Observa-se comportamento antagônico entre as variáveis VTP e Ds, o que evidencia a relação indireta entre elas, em que os maiores valores de Ds correspondem aos menores percentuais de porosidade. Nas áreas de SAF, tem-se os menores valores de Ds e, consequentemente, os maiores valores de VTP\%.

De maneira geral, pode-se visualizar tendência de agrupamento da área de figo $(4 \mathrm{I} / 4 \mathrm{~V})$ separando-se das demais. Isso está relacionado aos atributos químicos $\mathrm{P}$, $\mathrm{K}$ e COT. Na área com figo, observou-se os maiores valores desses nutrientes (Tabela 4), o que pode estar relacionado ao maior tempo de implantação da cultura (7 anos) e, também, às práticas de adubação verde e adição de estercos (bovino e de aviário), quando comparada às demais áreas. Assim, houve uma intensa reciclagem, e os nutrientes acumularam-se no solo, após a implantação da cultura.

A área do SAF tendeu a ficar sempre do lado esquerdo do gráfico (Figura 1), em correlação mais com o VTP. Na profundidade de 5-10 cm, essa área apresentou maiores valores de porosidade que as demais, nas duas estações (Tabela 2), o que reflete a influência das propriedades físicas do solo (Ds e VTP) nesse sistema, uma vez que essa área não foi submetida à influência antrópica e, portando, pode ser considerada mais estável, quando comparada às demais.

Theodoro et al. (2003) avaliaram as alterações nas propriedades edáficas de um Latossolo Vermelho, submetido ao manejo orgânico, convencional e em conversão da cultura do café, e ao compará-lo a um fragmento de mata nativa, observaram, por meio dos valores médios de $\mathrm{Al}$ e agregação do solo, que a área de manejo orgânico separou-se das demais formas de manejo. Apesar de não terem sido verificados elevados valores de $\mathrm{Al}$ na área de $\mathrm{SAF}$, foi observada melhoria na agregação do solo, constatada pelos maiores valores de DMP e VTP. Portanto, o SAF pode ser considerado similar à mata nativa. Theodoro et al. (2003) também observaram que o manejo orgânico distinguiu-se das demais formas de manejo, pelas propriedades químicas, em que se destacaram os valores de P e K. Resultado similar foi verificado no presente trabalho, em que elevados valores de $\mathrm{P}$ e $\mathrm{K}$ foram observados na área de figo (Tabela 4), provavelmente, por efeito da reciclagem de nutrientes promovida pelas folhas da figueira na superfície do solo, principalmente em sistemas agroecológicos.

\section{Conclusões}

1. O sistema maracujá/Desmodium propicia maiores valores de diâmetro médio ponderado e diâmetro médio geométrico, na camada superficial do solo, na estação chuvosa.

2. O sistema plantio direto apresenta maior agregação do solo, e o sistema agroflorestal maiores percentuais de porosidade total, nas duas estações, na camada de 5-10 $\mathrm{cm}$ de profundidade.

3. Aárea cultivada com figo está associada a maiores índices de fertilidade do solo.

\section{Referências}

ALTIERI, M.; NICHOLLS, C.I. Agroecologia: teoría práctica para una agricultura sustentable. México, DF: PNUMA, 2000. 250p.

ARAUJO, R.; GOEDERT, W.J.; LACERDA, M.P.C. Qualidade de um solo sob diferentes usos e sob cerrado nativo. Revista Brasileira de Ciência do Solo, v.31, p.1099-1108, 2007.

BERTOL, L.; ALBUQUERQUE, J.A.; LEITE, D.; AMARAL, A.J.; ZOLDAN JUNIOR, W.A. Propriedades físicas do solo sob preparo convencional e semeadura direta em rotação e sucessão de culturas, comparadas às do campo nativo. Revista Brasileira de Ciência do Solo, v.28, p.155-163, 2004.

CARDOZO, S.V.; PEREIRA, M.G.; RAVELli, A.; LOSS, A. Caracterização de propriedades edáficas em áreas sob manejo orgânico e natural na região serrana do Estado do Rio de Janeiro. Semina: Ciências Agrárias, v.29, p.517-530, 2008.

CASTRO, C.M.; ALMEIDA, D.L.; RIBEIRO, R.L.D. No-tillage, green manure and supplementation with poultry manure on organic eggplant production. Pesquisa Agropecuária Brasileira, v.40, p.495-502, 2005. 
CESAR, M.N.Z. Desempenho de duas cultivares de pimentão (Capsicum annuum L.) em sistema orgânico de produção, submetidas a desbaste de ramos e consorciadas com Crotalaria. 2004. 57p. Dissertação (Mestrado) - Universidade Federal Rural do Rio de Janeiro, Seropédica.

DE-POLLI, H.; ALMEIDA, D.L.; SANTOS, G.A.; CUNHA, L.H.; FREIRE, L.R.; AMARAL SOBRINHO, N.M.B.; PEREIRA, N.N.C.; EIRA, P.A.; BLOISE, R.M.; SALER, R.C. Manual de adubação para o Estado do Rio de Janeiro. Itaguaí: Editora da Universidade Rural, 1988.

DUDA, G.P.; GUERRA J.G.M.; MONTEIRO, M.T.; DE-POLLI, H. Perennial herbaceous legumes as live soil mulches and their effects on C, N and P of the microbial biomass. Scientia Agricola, v.60, p.139-147, 2003.

EBERHARDT, D.N.; VENDRAME, P.R.S.; BECQUER, T.; GUIMARÃES, M.F. Influência da granulometria e da mineralogia sobre a retenção de fósforo em Latossolos sob pastagens no Cerrado. Revista Brasileira de Ciência do Solo, v.32, p.1009-1016, 2008.

EMBRAPA. Centro Nacional de Pesquisa de Solos. Manual de métodos de análise de solo. 2.ed. Rio de Janeiro, 1997. 212p. (Embrapa-CNPS. Documentos, 1).

EMBRAPA SOLOS. Sistema Brasileiro de Classificação de Solos. 2.ed. Rio de Janeiro, 2006. 306p.

ESPINDOLA, J.A.A. Avaliação de leguminosas herbáceas perenes usadas como cobertura viva de solo e seus efeitos sobre a produção da bananeira (Musa spp.). 2001. 144p. Tese (Doutorado) - Universidade Federal Rural do Rio de Janeiro, Seropédica.

GOMES, J.B.V.; CURI, N.; MOTTA, P.E.F.; KER, J.C.; MARQUES, J.J.G.S.M.; SCHULZE, D.G. Análise de componentes principais de atributos físicos, químicos e mineralógicos de solos do bioma Cerrado. Revista Brasileira de Ciência do Solo, v.28, p.137-153, 2004.

HERLIHY, M.; McCARTHY, J. Association of soil-test phosphorus with phosphorus fractions and adsorption characteristics. Nutrient Cycling in Agroecosystems, v.75, p.79-90, 2006.

LOSS, A. Frações orgânicas e agregação do solo em diferentes sistemas de produção orgânico. 2008. 73p. Dissertação (Mestrado) - Universidade Federal Rural do Rio de Janeiro, Seropédica.

PERIN, A.; GUERRA, J.G.M.; TEIXEIRA, M.G.; PEREIRA, M.G.; FONTANA, A. Efeito da cobertura viva com leguminosas herbáceas perenes na agregação de um Argissolo. Revista Brasileira de Ciência do Solo, v.26, p.713-720, 2002.

PINHEIRO, E.F.M.; PEREIRA, M.G.; ANJOS, L.H.C. Aggregates distribution and soil organic matter under different tillage system for vegetable crops in a Red Latosol from Brazil. Soil Tillage Research, v.77, p.79-84, 2004.

REICHERT, J.M.; LIMA, C.L.R.; DALMOLIN, R.S.D.; REINERT, D.J.; GONÇALVES, C.S.; NUNES, M.L. Agregação de um Planossolo sistematizado há um ano e sob cultivo de arroz irrigado. Ciência Rural, v.36, p.837-844, 2006.

REINERT, D.J.; REICHERT, J. Propriedades físicas de solos em sistema plantio direto irrigado. In: CARLESSO, R.; PETRY, M.; ROSA, G.; CERETTA, C.A. Irrigação por Aspersão no Rio Grande do Sul. Santa Maria, 2001. p.114-131.

RIBAS, R.G.T.; JUNQUEIRA, R.M.; OLIVEIRA, F.L.; GUERRA, J.G.M.; ALMEIDA, D.L.; ALVES, B.J.R.; RIBEIRO, R.L.D. Desempenho do quiabeiro (Abelmoschus esculentus) consorciado com Crotalaria juncea sob manejo orgânico. Agronomia, v.37, p.80-84, 2003.

SAS INSTITUTE. SAS/STAT User's guide, Version 8. Cary: SAS Institute, 1999.

SILVA, C.F.; LOSS, A.; SILVA, E.M.R.; PEREIRA, M.G.; CORREIA, M.E.F. Alterações químicas e físicas em áreas de agricultura no entorno do parque Estadual da Serra do Mar, Ubatuba, SP. Revista de Ciências Agrárias, v.46, p.9-28, 2006.

SILVA, E.E. Manejo orgânico da cultura da couve em rotação com o milho, consorciados com leguminosas para adubação verde intercalar em plantio direto. 2006. 57p. Dissertação (Mestrado) - Universidade Federal Rural do Rio de Janeiro, Seropédica.

SILVEIRA NETO, A.N.; SILVEIRA, P.M.; STONE, L.F.; OLIVEIRA, L.F.C. Efeitos de manejo e rotação de culturas em atributos físicos do solo. Pesquisa Agropecuária Tropical, v.36, p.1-20, 2006.

SISTI, C.P.J.; SANTOS, H.P.; KOHHANN, R.A.; ALVES, B.J.R.; URQUIAGA, S.; BODDEY, R.M. Change in carbon and nitrogen stocks in soil under 13 years of conventional or zero tillage in southern Brazil. Soil \& Tillage Research, v.76, p.39-58, 2004.

SOUSA NETO, E.L.; ANDRIOLI, I.; BEUTLER, A.N.; CENTURION, J.F. Atributos físicos do solo e produtividade de milho em resposta a culturas de pré-safra. Pesquisa Agropecuária Brasileira, v.43, p.255-260, 2008.

TARRÉ, R.; MACEDO, R.; CANTARURI, R.B.; REZENDE, C.P.; PEREIRA, J.M.; FERREIRA, E.; ALVES, B.J.R.; URQUIAGA, S.; BODDEY, R.M. The effect of the presence of legume on a Brachiaria humidicola pasture. Plant and Soil, v.234, p.15-26, 2001.

THEODORO, V.C.A.; ALVARENGA, M.I.N.; GUIMARÃES, R.J.; SOUZA, C.A.S. Alterações químicas em solo submetido a diferentes formas de manejo do cafeeiro. Revista Brasileira de Ciência do Solo, v.27, p.1039-1047, 2003.

TINO, V.F. Utilização da análise de componentes principais para a regulagem de máquinas de injeção plástica. 2005. Dissertação (Mestrado) - Universidade Federal do Rio de Janeiro, Rio de Janeiro.

WENDLING, B.; JUCKSCH, I.; MENDONÇA, E.S.; NEVES, J.C.L. Carbono orgânico e estabilidade de agregados de um Latossolo Vermelho sob diferentes manejos. Pesquisa Agropecuária Brasileira, v.40, p.487-494, 2005.

Recebido em 26 de fevereiro de 2008 e aprovado em 8 de dezembro de 2008 